\title{
¿A dónde van los expresidentes latinoamericanos? Salida de la política y trayectorias pospresidenciales en América Latina (1978-2018) ${ }^{*}$
}

\author{
Cristian Márquez Romo (México)**
}

\section{Resumen}

La mayor parte de los estudios sobre trayectorias políticas centran su atención en la entrada o el desarrollo de estas. Los trabajos sobre la salida de la política, en general, y en el caso de los presidentes, en particular, son prácticamente inexistentes. Este trabajo tiene como propósito analizar las trayectorias de los expresidentes latinoamericanos a partir de dos estrategias metodológicas: el análisis estadístico y la revisión documental. Para ello, se elabora una clasificación tipológica, reconstruyendo las trayectorias de los expresidentes de dieciocho países latinoamericanos desde las transiciones a la democracia hasta el presente (1978-2018), partiendo de la premisa de que los expresidentes latinoamericanos tienden a seguir su carrera política al dejar el cargo. Si bien hay determinados rasgos objetivables que diferencian a los expresidentes que se desempeñan en el sector público o abandonan la vida pública de aquellos que lo hacen en la esfera privada, esta última no representa un impedimento para seguir teniendo presencia política.

\section{Palabras clave}

Comportamiento Político; Administración Pública; Élites Políticas; Pospresidencia; Capital Político; América Latina.

Fecha de recepción: octubre de 2018

- $\quad$ Fecha de aprobación: marzo de 2019

\section{Cómo citar este artículo}

Márquez Romo, Cristian. (2019). ¿A dónde van los expresidentes latinoamericanos? Salida de la política y trayectorias pospresidenciales en América Latina (1978-2018). Estudios Políticos (Universidad de Antioquia), 55, pp. 37-60. http://doi.org/10.17533/udea.espo.n55a03

\footnotetext{
* Este artículo fue elaborado en el marco del Máster en Estudios Latinoamericanos de la Universidad de Salamanca, España, bajo la dirección de Manuel Alcántara Sáez. Les agradezco tanto a él como a Mélany Barragán y a Francisco Sánchez por su disposición para ceder la base de datos de presidentes latinoamericanos, así como a los tres revisores anónimos de esta revista por sus comentarios.

** Politólogo. Miembro del Instituto de Iberoamérica, Universidad de Salamanca, España. Correo electrónico: cmarquez@usal.es
} 


\title{
Where do Latin American Former Presidents Go? The Way Out of Politics and Post-Presidential Careers in Latin America (1978-2018)
}

\begin{abstract}
Empirical studies based on the careers of Latin American former presidents are practically non-existent. Most of the literature is focused on the development of the political career. Hence, the main goal of this proposal is to fill in this gap. It presents an empirical analysis based on the post-presidencies of eighteen Latin American countries since their transitions to democracy in 1978 up until 2018 in order to explore relevant factors that explain them. Using statistical and documentary methods, we create a typology of post-presidential careers, considering variables related to the individual characteristics of the former presidents, as well as with their previous political careers. The study found that former presidents tend to continue their political careers after their presidential period is over. The study also showed that it is possible to assess differences between those who continue in the public life and those who abandon it; however, even if they retired from public life it does not represent an obstacle for them to keep having political influence.
\end{abstract}

\section{Keywords}

Political Behavior; Public Administration; Political Elites; Post-Presidency; Political Capital; Latin America. 
¿A dónde van los expresidentes latinoamericanos? Salida de la política y trayectorias...

\section{Introducción}

«Nada es tan peligroso como dejar permanecer largo tiempo a un mismo ciudadano en el poder. El pueblo se acostumbra a obedecerlo y él se acostumbra a mandar; de donde se origina la usurpación y la tiranía» (Restrepo, 1945, p. 75). Estas palabras fueron pronunciadas por Simón Bolívar en un discurso en el Congreso de Angostura, previo a dejar el gobierno. Del mismo modo, el primer presidente de Estados Unidos, George Washington, se enfrentó a un dilema similar cuando estaba a punto de convertirse en el primer presidente del país norteamericano en dejar el cargo. El historiador Joseph Ellis (2004, p. 104) relata cómo ante la sospecha de quienes creían que Washington seguiría gobernando, transformándose en monarca, Jorge III afirmó que si resistía las tentaciones de mantenerse en el cargo se convertiría en «el mejor hombre del mundo».

El modelo presidencialista latinoamericano tuvo como referencia el sistema político estadounidense, inspirado en la famosa frase de John Adams primer vicepresidente y segundo presidente de Estados Unidos—, «un gobierno de leyes y no de hombres» (Anderson, 2010, p. 67). De hecho, en el largo debate iniciado por Juan J. Linz (2013) hace tres décadas, quien confrontaba los vicios del presidencialismo con las virtudes del parlamentarismo, en un momento en que se discutía las posibilidades de que las democracias latinoamericanas lograran su consolidación, argumentó que el caso estadounidense era una excepción: «la única democracia presidencial con una larga historia de continuidad constitucional es la de los Estados Unidos» (p. 12). No obstante, poco se suele decir acerca de los problemas que tuvo el caso estadounidense para frenar el ímpetu reeleccionista, así como la pulsión de los mandatarios por permanecer en el cargo. Finalmente, la enmienda constitucional que limita a dos mandatos el periodo presidencial —el cual se mantiene hasta la actualidad - fue consecuencia de un largo debate iniciado luego de que Franklin D. Roosevelt fuera elegido por cuatro veces consecutivas.

En el fondo, se trata de una cuestión tan simple de entender como difícil de conseguir, que Seymour Martin Lipset resumió en una frase: «las normas democráticas requieren de la voluntad de los gobernantes para aceptar la derrota, dejar el cargo luego de perder una elección, seguir las reglas incluso cuando vayan en contra de sus propios intereses» (Anderson, 2010, p. 64). Aceptar la derrota, asumir que se tiene que dejar el poder después de un 
tiempo determinado, es uno los activos más valiosos de la política democrática, en contraposición con otro tipo de regímenes - teocráticos, autocráticos y monárquicos-: aquellos en los que los gobernantes ocupan cargos de forma arbitraria, a través de la fuerza o a partir de elecciones en las cuales, previo al inicio del periodo electoral, la suerte está echada y el vencedor del escrutinio no es nunca una sorpresa (Rouquié, 2011).

En Latinoamérica los liderazgos presidenciales estuvieron históricamente acompañados de una notoria ausencia de controles y un mayor poder que, por ingeniería institucional y cultura política, ha tenido la figura presidencial (Serrafero, 2011, p. 228). Por tanto, dejar el cargo, como un atributo sine qua non para la continuidad de un régimen democrático, representa uno de los mayores retos para democracias jóvenes en las cuales ocupar la presidencia de forma arbitraria se hizo rutinariamente por décadas.

Con todo, como han demostrado las once elecciones presidenciales celebradas durante el bienio 2014-2015, no sin sobresaltos, las elecciones y la alternancia se han ido normalizando en la región. Hoy, por lo menos en el ámbito nacional, los procesos electorales son rutina sin precedentes en la historia (Alcántara y Tagina, 2016). Y si las elecciones son una condición

[ 40 ] necesaria mas no suficiente para que un régimen pueda ser considerado democrático (Dahl, 2010; Levitsky y Way, 2012), resulta entendible que, dado el complejo histórico latinoamericano, basado en la violación frecuente de la práctica electoral, la preocupación electoral llenara por completo la agenda de la democratización (Alcántara, 2016), propiciando, al mismo tiempo, un mayor interés en reformar el acceso al poder que a su ejercicio (Merino, 2003).

Aunque históricamente la evidencia apunta a que la tendencia al conflicto aumenta a medida en que un sistema político se acerca al personalismo y disminuye cuando las instituciones son fuertes, los impulsos personales quedan sometidos a ellas y las normas que regulan la sucesión son claras (Sribman, 2014, p. 3), en regímenes como los latinoamericanos, los cuales institucionalizaron la sucesión a partir de medios no democráticos, asegurar la autonomía del presidente entrante y la ausencia del saliente en un entorno en el cual la aspiración mayoritaria de los políticos es «seguir en política» sugiere algunas contradicciones. Por tanto, pese a que se trata de una herramienta indispensable para la rotación de las élites políticas, así 
¿A dónde van los expresidentes latinoamericanos? Salida de la política y trayectorias...

como un elemento fundamental en términos de transparencia y rendición de cuentas exigible a cualquier sistema político democrático (Alcántara, 2013), el estudio sistemático de la salida de la política, en general, y las trayectorias de los expresidentes, en particular, ha sido una cuestión que ha pasado desapercibida, tanto en la academia como en la opinión pública.

A cuatro décadas de las transiciones a la democracia, tanto en Europa como América Latina la cifra de expresidentes seguirá aumentando, debido al incremento del número de países democráticos, por un lado, y a las expectativas de vida, por el otro (Anderson, 2010). Por consiguiente, la necesidad de discutir a dónde van y en qué se desempeñan los expolíticos es una cuestión que cobrará cada vez mayor relevancia. Integrada como un indicador de la vida política del sistema del cual el individuo formó parte, la salida de la política puede arrojar indicadores para: a) validar una carrera profesional; b) desmentir o confirmar el designio inicial del sujeto; c) aportar información acerca de las razones que llevan a un político a abandonar su actividad y de las actividades que realiza inmediatamente después del ejercicio de lo público; d) explicar el papel que ejercen en la esfera pública los políticos retirados; y finalmente, e) explicar los medios a través de los cuales rentabilizan o no, con los propios mecanismos de salida, el capital obtenido a lo largo de su carrera (Alcántara, 2012).

Adicionalmente, puede contribuir a la discusión normativa acerca de la necesidad de establecer o no límites a la rentabilización del capital político — pasando por el estudio de las «puertas giratorias» (Barragán, 2016a)—, los vínculos entre las élites políticas y las élites empresariales —las redes de interlocking directorates (Cárdenas, 2016)—, la organización de las élites y el poder —la «captura política» (Crabtree y Durand, 2017)—y la ambición (Schlesinger, 1966) en la vida pospresidencial.

Los trabajos empíricos sobre la salida de la política y las trayectorias pospresidenciales son prácticamente inexistentes. La mayor parte de los estudios sobre carreras políticas centran su atención en la entrada o desarrollo de estas (Siavelis y Morgenstern, 2008; Samuels y Shugart, 2010; Joignant, 2012). Hay escasos estudios sobre su retiro, la forma en que los expolíticos invierten el capital acumulado durante su carrera, las razones por las que dejan la política o el lugar a donde van luego de hacerlo (Alcántara, 2012). 
Este artículo forma parte de una línea de investigación más amplia (Alcántara, Barragán y Sánchez, 2016; Alcántara, Blondel y Thiébault, 2018), cuya base de datos fue construida por Manuel Alcántara, Mélany Barragán y Francisco Sánchez, y facilitada en el marco del Máster en Estudios Latinoamericanos de la Universidad de Salamanca para el proceso de investigación. La principal fuente consultada para obtener información relativa a las trayectorias de los expresidentes ha sido el servicio de información Biografías Líderes Políticos del Barcelona Centre for International Affairs (Cidob, s. f.), complementada por fuentes bibliográficas y hemerográficas.

El principal objetivo de este trabajo es contribuir a llenar un vacío a través de un doble propósito: analizar empíricamente el itinerario que han seguido los expresidentes latinoamericanos durante el intervalo histórico posterior a las transiciones a la democracia en la región, explorando factores de relevancia para explicar dicho comportamiento. La pregunta que dirige este artículo es la siguiente: iqué factores explican las distintas trayectorias pospresidenciales de los expresidentes latinoamericanos, desde las transiciones a la democracia hasta el presente? Con el propósito de dar respuesta a este interrogante, se construye una tipología de trayectorias pospresidenciales que permite dar cuenta del itinerario que siguen los expresidentes al dejar el cargo, a partir de tres dimensiones: a) público, b) privado-no gubernamental y c) abandono de la vida pública. Posteriormente, se explora qué factores son relevantes para explicar las trayectorias pospresidenciales, considerando variables de diversa índole, a partir de tres dimensiones: a) sociodemográficas, b) institucionales y c) de trayectoria política-estructura de oportunidad.

A lo largo del trabajo se recurre al concepto «salida de la política», con base en el modelo para el estudio de la carrera política de Manuel Alcántara (2012, pp. 129-133). Al aplicarlo al análisis de la expresidencia en dieciocho países latinoamericanos, se considera el momento en el que los presidentes dejan el cargo y en qué circunstancia —es decir, el «mecanismo de salida»—, ${ }^{1}$ así como la principal actividad en la cual se desempeñan profesionalmente luego de hacerlo. A partir de ello se operacionaliza el concepto «trayectoria pospresidencial».

\footnotetext{
${ }^{1}$ Por mecanismo de salida se contemplan cinco escenarios: a) derrota electoral; b) voluntario-renuncia; c) reelección no permitida; d) inhabilitación; y e) fallecimiento.
} 


\section{El estudio de las élites políticas}

Desde los primeros trabajos de Gaetano Mosca en 1884, Wilfredo Pareto en 1901, Robert Michels en 1911, Max Weber en 1918 y más tarde Wright Mills en 1956, el estudio de las élites políticas ha constituido uno de los ámbitos de estudio tradicionales de las Ciencias Sociales. ${ }^{2}$ Como subraya Mélany Barragán (2016b, p. 47), las élites que ejercen el poder político se han estudiado a partir de su perfil sociodemográfico (Mills, 1956), de los procesos de socialización y profesionalización política (Hyman, 1959; Schmitter, 2010; Cabezas y Barragán, 2014), del rol ejercido por la ambición (Schlesinger, 1966) o del vínculo entre la calidad de los políticos y la calidad de la democracia (Morlino, 2014; Alcántara, Barragán y Sánchez, 2016). No obstante, existe un vacío en la literatura acerca de las élites políticas que dejan la esfera pública para desempeñarse en otros ámbitos.

El paradigma de la política como profesión se dio a partir del surgimiento del Estado moderno. Weber (1956 pp. 92-93) introduce en de su tipología de políticos profesionales ${ }^{3}$ la distinción entre vivir «de» la política y «para» la política. Así, la profesionalización de la política se produce por tres elementos que se entrelazan: a) personas con ambición, b) el desarrollo de las instituciones y c) apatía de la ciudadanía para intervenir en lo público (Alcántara, 2012, p. 21). La política puede ser considerada una profesión, en tanto se trata, como todas las demás, de una actividad remunerada y que tiende a ser de tiempo completo, que obliga a los individuos a hacer usufructo de distintos recursos — materiales y simbólicos-.

Así, si entender el rol desempeñado por la ambición resulta clave para analizar el desarrollo de la carrera política, lo son igualmente los medios y estrategias para hacerlo, en tanto se trata, en última instancia, de instrumentos utilizados para ejercer el poder político. De ahí que, el actor político pueda ser un reflejo del contexto sociopolítico en el cual desarrolla su carrera, las dinámicas de poder y las pautas de interacción entre los distintos actores

\footnotetext{
${ }^{2}$ Con base en la literatura que recorre desde los teóricos elitistas hasta el marxismo, en este artículo se considera «élite política» a aquellos individuos que concentran y detentan el poder político, incluyendo a los políticos, los grupos de presión y las organizaciones políticas.

${ }^{3}$ Por político profesional, Weber (1956, pp. 92-93) considera individuos que se dedican a la política a tiempo completo, como posteriormente lo hizo Giovanni Sartori (1992, p. 178) y más tarde Manuel Alcántara (2012, p. 22).
} 
(Barragán, 2015). En las democracias representativas el capital político ${ }^{4}$ contiene una serie de activos a partir de los cuales las élites políticas buscan presentarse como legitimas para ejercer la dominación. Puesto que a partir de la expansión de los partidos de masas y de la expansión de la democracia representativa la entrada de las élites en política se da ya sea a través de procesos electorales o de fórmulas de designación, estos elementos son relevantes para analizar el tipo de carrera política.

En el caso de los expresidentes latinoamericanos, hay quienes llegaron a la presidencia luego de una larga carrera institucional, cuya entrada fue a partir de cargos de elección o designación; otros, por el contrario, lo hicieron sin haber ocupado ningún cargo previamente, denominados outsiders. Asimismo, hay quienes desarrollaron una carrera partidista a partir de una determinada combinación de cargos en distintos niveles y con un capital fundamentalmente político; así como quienes, por el contrario, lo hicieron utilizando un capital técnico, derivado de la popularidad de su ocupación previa.

Este trabajo parte de la premisa de que la confluencia de estos elementos personales e institucionales influyen en la trayectoria que desarrollan los jefes de Estado al dejar el cargo. La perspectiva sobre cómo los políticos en [ 44 ] general y los jefes de Estado en particular ${ }^{5}$ invierten su capital durante su trayectoria pospresidencial puede arrojar puntos de contraste entre países, así como patrones de comportamiento entre los tipos de capital y su relación con nichos en los cuales se desempeñan los expresidentes.

\section{La pospresidencia y los mecanismos de salida}

Desde 1970 alrededor de 1160 individuos en el mundo han ocupado el cargo de jefe de Estado o de Gobierno en aproximadamente 190 países; de ellos, alrededor de 30 han sido monarcas — es decir, no tienen que abandonar el cargo—; 85 han muerto en funciones; alrededor de 115 han sido depuestos

\footnotetext{
${ }^{4}$ Por capital político se entiende: «los recursos de los que disponen los individuos en distintos momentos del tiempo que les permiten tener acceso a una posición, emprender una carrera parlamentaria o gubernamental (o una combinación de ambas) y, por tanto, acumular lo que se suele vagamente calificar de poder político» (Joignant, 2012, pp. 589-90). Como parte del capital político se aborda la tipificación del capital original, basado en cinco subtipos: a) político, b) económico, c) familiar, d) técnico y e) notoriedad (Alcántara 2012, p. 133).

${ }^{5}$ La Presidencia conlleva una serie de ventajas para el ocupante del cargo, desde reconocimiento y visibilidad pública, acceso a los recursos y fuentes de financiamiento gubernamentales, el despliegue de las relaciones públicas que establece la Presidencia con los sectores públicos y privados del país y del extranjero, entre otros (Serrafero, 2011).
} 
¿A dónde van los expresidentes latinoamericanos? Salida de la política y trayectorias...

por acciones militares —o invasiones—; y 190 continúan en el cargo. Así, puesto el énfasis en los primeros ministros y presidentes democráticamente electos, ello sugiere que a lo largo de las últimas cuatro décadas alrededor de 700 líderes políticos se han enfrentado, en el momento culmen de su carrera, al dilema de abandonar el cargo, así como al de en qué ocuparse luego de hacerlo (Grossman citado en Anderson, 2010, p. 65).

Al analizar la experiencia de treinta y cinco presidentes estadounidenses, Anderson (2010) observó cómo ciertos elementos se mezclan con el hecho de que, al hacerlo, muchos pierden la orientación acerca de cómo regresar a vivir una vida ordinaria. Y aunque contar con los medios básicos de subsistencia fue una preocupación que estuvo presente desde Washington, previo a que existiera pensión para expresidentes, es posible también observar preocupaciones comunes en el ámbito posmaterial, como proteger su legado en términos políticos y simbólicos. Con todo, hoy las circunstancias en las que viven los expresidentes han cambiado. Además de que la pensión se ha generalizado, desde el último tercio del siglo xx paulatinamente los exmandatarios han comenzado a caer en cuenta de que, con el surgimiento de los medios masivos y el avance de la globalización, el prestigio que otorga la Presidencia puede ser rentable. Así, a pesar de que tanto en Europa como en Estados Unidos y América Latina suelen tener una pensión que les permite vivir muy por encima de la mayoría de los ciudadanos, se ha vuelto común que los expresidentes busquen otras fuentes de financiamiento. ${ }^{6}$

En América Latina, a cuatro décadas de las transiciones a la democracia, muchos expresidentes con salud y una larga vida por delante han buscado los medios para desarrollar una carrera pospresidencial. ${ }^{7}$ De ahí que, entender

\footnotetext{
${ }^{6}$ Vender sus memorias, incorporarse a universidades, crear fundaciones, impartir conferencias, desempeñarse en lobbies y en consejos de administración. En la actualidad, en cualquier país democrático las probabilidades de que los políticos creen una expresidencia activa son cada vez mayores; por tanto, mayores son las probabilidades que tienen de rentabilizar el capital político que otorga la presidencia durante su trayectoria posterior (Vaughn, 2015; Clark, 2015).

${ }^{7}$ Hay una larga lista de expresidentes que tuvieron o siguen teniendo presencia e influencia en la opinión pública: Raúl Alfonsín, Eduardo Duhalde, Carlos Menem, Néstor Kirchner y Cristina Fernández en Argentina; Carlos Mesa y Jorge Quiroga en Bolivia; Fernando Henrique Cardoso, Lula da Silva y Dilma Rousseff en Brasil; Ricardo Lagos, Patricio Aylwin, Eduardo Frei, Michelle Bachelet y Sebastián Piñera en Chile; Óscar Arias y Laura Chinchilla en Costa Rica; Daniel Ortega, Violeta Barrios de Chamorro y Arnoldo Alemán en Nicaragua; Óscar Berger y Álvaro Arzú en Guatemala; Carlos Flores Facussé en Honduras; Rodrigo Borja y Rafael Correa en Ecuador; Carlos Salinas, Ernesto Zedillo y Felipe Calderón en México; Leonel Fernández en República Dominicana; Andrés Pastrana y Álvaro Uribe en Colombia; y Julio María Sanguinetti, José Mujica y Tabaré Vázquez en Uruguay.
} 
la pospresidencia como el final de la carrera política representa un error. Esta es, fundamentalmente, una continuación de la carrera política y, como tal, debería ser transparente, en tanto puede ser garante de un ejercicio político de mayor calidad (Alcántara, 2012), así como una fuente de información acerca de los incentivos que pueden tener los expresidentes antes y después de dejar el cargo.

\section{Metodología del análisis}

Dentro de las Ciencias Sociales hay tres métodos para teorizar y realizar proposiciones de carácter empírico: experimentales, estadísticos y comparativos (Pasquino, 2011; Sartori, 2002). Un rasgo distintivo de los trabajos politológicos, establecido como un procedimiento analítico central en la disciplina desde la década de 1970, es la utilización del método comparado como un instrumento apropiado para investigaciones en las cuales el número de casos es demasiado pequeño para permitir la utilización del análisis estadístico (Lijphart citado en Pérez, 2010, p. 126). Con 119 expresidentes que han ocupado el cargo de jefe de Estado en 18 países latinoamericanos a lo largo de cuatro décadas como unidad de análisis, este trabajo utiliza un abordaje estadístico, complementado por una revisión documental (Cidob, s.

[ 46 ] f.). Para ello se construye una tipología para indagar cómo son las trayectorias de los expresidentes latinoamericanos, con el objetivo de explorar qué variables intervienen en las trayectorias. Así, identificar los distintos itinerarios que tienen los expresidentes latinoamericanos en relación con variables de distinta índole, puede ser de utilidad para explorar los incentivos con que cuentan los expresidentes, así como para contrastar los tipos de trayectorias pospresidenciales existentes y la relevancia explicativa de cada una de las variables y dimensiones (véase cuadro 1).

Cuadro 1. Tipología de las trayectorias de los expresidentes latinoamericanos.

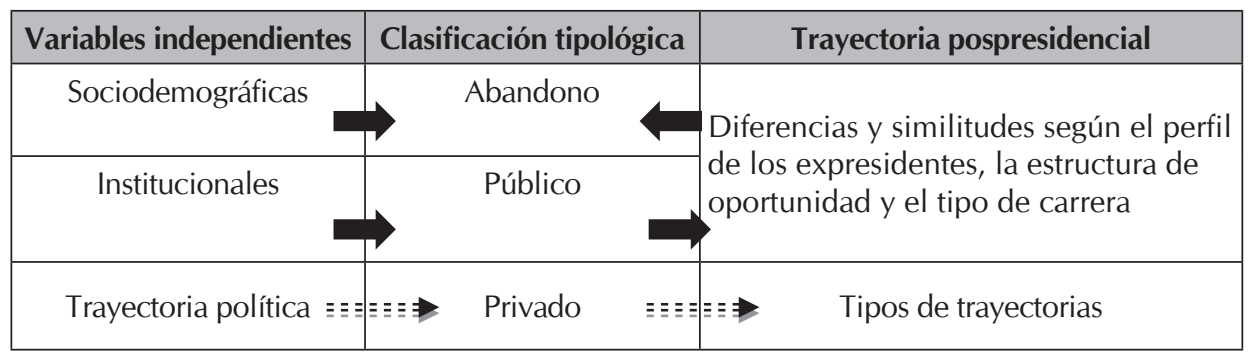

Fuente: elaboración propia. 
¿A dónde van los expresidentes latinoamericanos? Salida de la política y trayectorias...

La tipología incluye tres dimensiones: a) público-vinculación política, b) privado-no gubernamental y c) abandono de la vida pública (véase cuadro 2).

Cuadro 2. Trayectoria pospresidencial.

\begin{tabular}{|c|c|c|}
\hline Público-vinculación política & $\begin{array}{c}\text { Sector privado-no } \\
\text { gubernamental }\end{array}$ & Abandono \\
\hline $\begin{array}{c}\text { Cargo partidario } \\
\text { (Organizaciones vinculadas a los partidos) }\end{array}$ & Empresas transnacionales & Exilio \\
\hline $\begin{array}{c}\text { Público administrativo } \\
\text { (funcionario, embajador) }\end{array}$ & Empresas nacionales & $\begin{array}{c}\text { Enjuiciamiento y } \\
\text { abandono }\end{array}$ \\
\hline $\begin{array}{c}\text { Mandato Legislativo } \\
\text { (diputado, senador, concejal) }\end{array}$ & $\begin{array}{c}\text { Organizaciones no } \\
\text { gubernamentales }\end{array}$ & Mubilación \\
\hline $\begin{array}{c}\text { Mandato ejecutivo } \\
\text { (gobernador, alcalde, ministro) }\end{array}$ & Fundaciones \\
\hline $\begin{array}{c}\text { Organizaciones supranacionales } \\
\text { (ONU, BID, BM) }\end{array}$ & Think tanks & \\
\hline
\end{tabular}

Fuente: elaboración propia.

Las variables independientes están organizadas en torno a tres dimensiones:

a) Sociodemográfica. Las variables son: i) edad al dejar la presidencia,

ii) profesión, iii) género, iv) máximo nivel de estudios y v) capital original. ${ }^{8}$

b) Trayectoria política. Las variables son: i) canal de entrada, ii) primer cargo, iii) último cargo, iv) mecanismo de salida, v) socialización política familiar y vi) candidatura con/sin partido. ${ }^{9}$

c) Institucional. Las variables son: i) reelección y ii) tipo de salida. ${ }^{10}$

\footnotetext{
${ }^{8}$ En todos los casos se establece un margen de 5\% por categoría, creando una categoría residual para aquellas con un porcentaje menor.

${ }^{9}$ Por canal de entrada se considera si los expresidentes entraron en política a través de un cargo de elección o designación. Para primer cargo ocupado se crea una categoría residual para Ejecutivo regional (1,6\%), Legislativo regional (4,6\%), Legislativo local (4,7\%) y dirección institución pública; en el último cargo, para Poder Judicial $(0,8 \%)$, cargo militar $(0,8 \%)$, dirigencia de partido $(3,1 \%)$, Ejecutivo supranacional $(0,8 \%)$ y Ejecutivo local $(3,9 \%)$.

${ }^{10}$ La variable reelección no considera si el expresidente fue reelecto, sino si cuando este dejó el cargo existía la posibilidad de reelegirse posteriormente. Si existía la posibilidad de reelección consecutiva,
} 


\section{Análisis de datos}

Al clasificar los casos a nivel agregado es posible constatar que existen diferencias relevantes a lo largo del espectro público-privado-abandono (véase tabla 1). Por un lado, países cuyos exmandatarios se ubican en todos los casos dentro del sector privado — México-, así como quienes lo hacen en el sector público - Colombia y Panamá-; por otro, países que se inclinan en mayor medida hacia uno u otro sector; ${ }^{11}$ por último, los casos intermedios y aquellos que se inclinan más hacia el abandono. ${ }^{12}$

Argentina, Colombia y Panamá son tres países que ilustran la dimensión público-vinculación política. En el primer caso, desde Raúl Alfonsín hasta Cristina Fernández de Kirchner, todos los expresidentes, con excepción de Fernando de la Rúa, se han desempeñado en este sector (83,3\%). En el caso de Colombia y Panamá, todos los expresidentes. Los casos chileno y mexicano ilustran la dimensión privado-no gubernamental: en el primero, desde Patricio Aylwin hasta Sebastián Piñera, todos los exmandatarios, con excepción de Eduardo Frei y Michelle Bachelet, se han desempeñado en este sector (60\%); en el segundo, todos los exmandatarios. Expresidentes como Sebastián Piñera o Michelle Bachelet han utilizado la actividad profesional en la cual

[ 48 ] se desempeñaban como una etapa transitoria para regresar a la Presidencia, otros, como Ricardo Lagos, Eduardo Frei, Carlos Menem, Eduardo Duhalde y Lula da Silva, han tratado de hacerlo sin éxito. Por su parte, Vicente Fox, Felipe Calderón y Violeta Barrios de Chamorro han utilizado la expresidencia para seguir teniendo visibilidad en la esfera pública desde el sector privado. Finalmente, en sentido contrario de lo que sugiere la literatura sobre ambición, hay exmandatarios alejados del sector público para dedicarse a actividades privadas. ${ }^{13}$

pero el mandatario dejó el cargo, se contempla como «abandono voluntario». Todos los individuos que se enfrentaron a la prohibición de la reelección, sea absoluta — como en el caso mexicano y colombiano- o no consecutiva — como en el caso chileno o uruguayo-, son clasificados en el mecanismo de salida «reelección no permitida».

${ }^{11}$ Argentina (83,3\%), Guatemala (62,5\%), Brasil (66,7\%), Nicaragua (75\%), Paraguay (57,1\%) y Honduras $(44,4 \%)$ hacia el sector público; Chile (60\%), Uruguay (60\%) y Costa Rica $(50 \%)$ hacia el privado.

${ }^{12}$ Perú y Bolivia tienen 50\% público y 50\% abandono, y República Dominicana tiene 33,3\% en cada dimensión. Venezuela es el país con la mayor cantidad de casos de abandono (57,1\%), seguido por Perú (50\%), Bolivia (50\%) y Ecuador (38,5\%).

${ }_{13}$ Jorge Serrano Elías en Guatemala, Federico Franco en Paraguay o Hipólito Mejía Domínguez en República Dominicana. 
¿A dónde van los expresidentes latinoamericanos? Salida de la política y trayectorias...

Tabla 1. Trayectorias pospresidenciales en América Latina.

\begin{tabular}{|l|c|c|c|c|}
\hline \multicolumn{1}{|c|}{ País } & $\begin{array}{c}\text { Público } \\
\mathbf{\%} \mathbf{( N )}\end{array}$ & $\begin{array}{c}\text { Privado } \\
\mathbf{\%}(\mathbf{N})\end{array}$ & $\begin{array}{c}\text { Abandono } \\
\mathbf{\%}(\mathbf{N})\end{array}$ & $\begin{array}{c}\text { Total } \\
\mathbf{\%}(\mathbf{N})\end{array}$ \\
\hline Argentina & $83,3(5)$ & $0(0)$ & $16,7(1)$ & $100(6)$ \\
\hline Bolivia & $50(4)$ & $0(0)$ & $50(4)$ & $100(8)$ \\
\hline Chile & $40(2)$ & $60(3)$ & $0(0)$ & $100(5)$ \\
\hline Colombia & $100(7)$ & $0(0)$ & $0(0)$ & $100(7)$ \\
\hline Costa Rica & $12,5(1)$ & $50(4)$ & $37,5(3)$ & $100(8)$ \\
\hline Ecuador & $38,5(5)$ & $23,1(3)$ & $38,5(3)$ & $100(11)$ \\
\hline El Salvador & $50(3)$ & $16,7(1)$ & $33,3(2)$ & $100(6)$ \\
\hline Guatemala & $62,5(5)$ & $25(2)$ & $12,5(1)$ & $100(8)$ \\
\hline Honduras & $44,4(4)$ & $22,2(2)$ & $33,3(3)$ & $100(9)$ \\
\hline México & $0(0)$ & $100(3)$ & $0(0)$ & $100(3)$ \\
\hline Nicaragua & $75(3)$ & $25(1)$ & $0(0)$ & $100(4)$ \\
\hline Panamá & $100(5)$ & $0(0)$ & $0(0)$ & $100(5)$ \\
\hline Paraguay & $57,1(4)$ & $14,3(1)$ & $28,6(2)$ & $100(7)$ \\
\hline Perú & $50(3)$ & $0(0)$ & $50(3)$ & $100(6)$ \\
\hline República Dominicana & $33,3(2)$ & $33,3(2)$ & $33,3(2)$ & $100(6)$ \\
\hline Uruguay & $40(2)$ & $60(3)$ & $0(0)$ & $100(5)$ \\
\hline Venezuela & $42,9(3)$ & $0(0)$ & $57,1(4)$ & $100(7)$ \\
\hline Brasil & $66,7(4)$ & $33,3(2)$ & $0(0)$ & $100(6)$ \\
\hline & $\mathbf{5 2 , 5 ( 6 2 )}$ & $\mathbf{2 1 , 8 ( 2 6 )}$ & $\mathbf{2 6 , 1 ( 3 1 )}$ & $\mathbf{1 0 0}(\mathbf{1 1 9})$ \\
\hline
\end{tabular}

Fuente: elaboración propia.

Ahora, los resultados del análisis de correspondencias múltiples (ACA) dan cuenta de tres variables que arrojan mayor relevancia para explicar las trayectorias pospresidenciales: a) capital original, b) familia en política y c) máximo nivel de estudios (véase gráfica 1).

De los expresidentes que contaban con un capital político, 64,4\% se desempeñaron en el sector público y 64,3\% abandonaron la vida pública; en contraste, 33\% lo hicieron en el sector privado. Por su parte, quienes contaban con un capital familiar, 50\% se ubicaron en el sector privado, a diferencia de aquellos que lo hicieron en el sector público $(25,4 \%)$ o abandonaron la vida pública $(28,6 \%)$. 
Gráfica 1. Análisis de correspondencias múltiples: capital original, familia en política y máximo nivel de estudios.

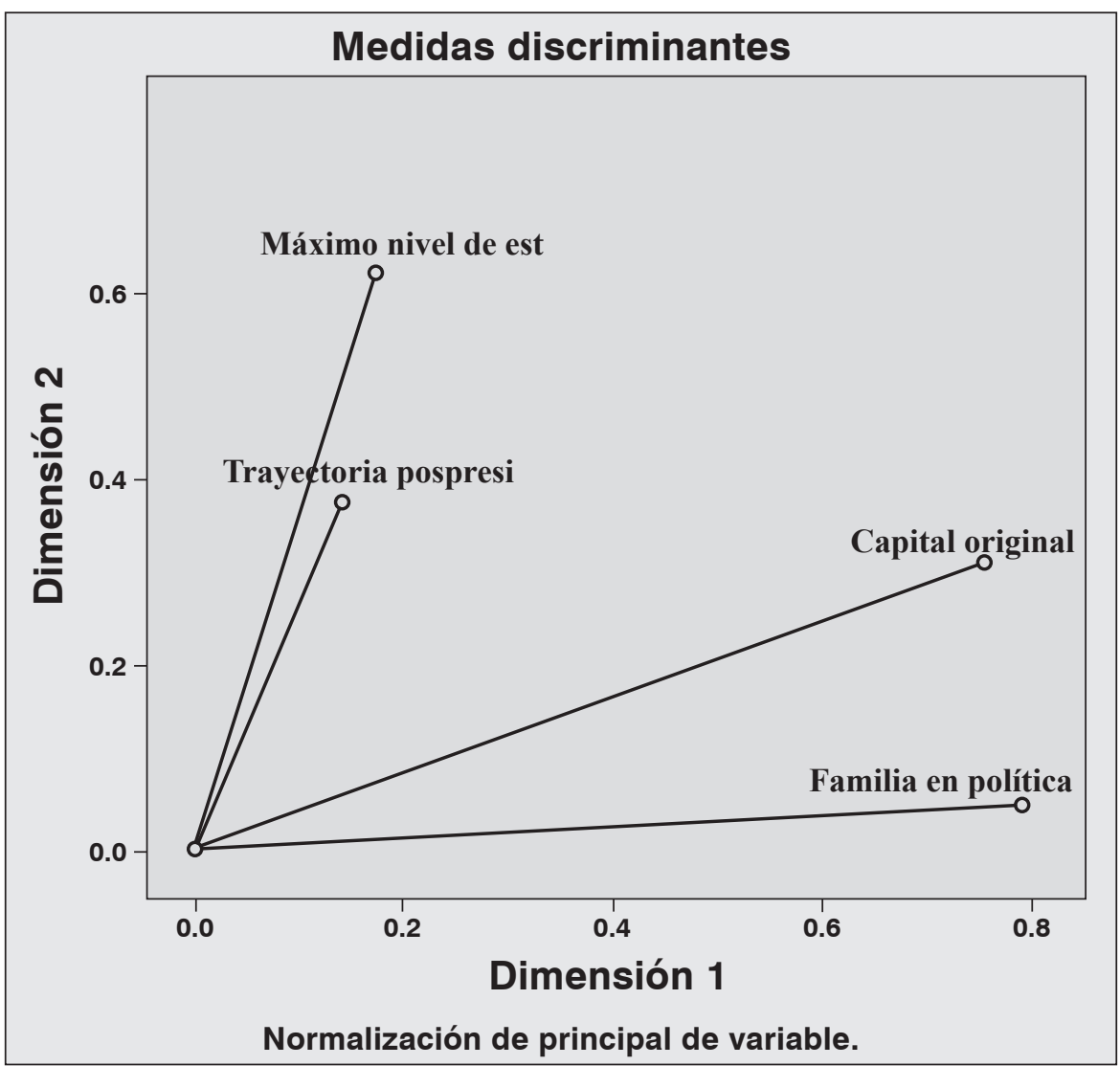

Fuente: elaboración propia.

Para aquellos mandatarios que contaban con un capital económico al llegar a la Presidencia se aprecian diferencias relevantes: hay una relación entre aquellos mandatarios que llegaron a la Presidencia con un capital político y familiar. Dentro del capital original, estos dos subtipos son significativos para el sector en el que se desempeñaron posteriormente los exmandatarios (véase tabla 2). Por consiguiente, al clasificar a los casos que contaban con familia en política (véase tabla 3), $60 \%$ de quienes se ubicaron en el sector privado dicen tener familia en política; en contraste con quienes afirmaron no tenerla y se ubicaron en el sector público (53,3\%), o abandonaron la vida pública (60\%). Esta tendencia se refuerza al observar el máximo nivel de estudios (véase tabla 4): por un lado, el 65\% de los exmandatarios con un nivel de 
¿A dónde van los expresidentes latinoamericanos? Salida de la política y trayectorias...

estudios superior al universitario se ubicaron en el sector privado, a diferencia de quienes lo hicieron en el sector público o abandonaron la vida pública. En otras palabras, quienes cuentan con estudios universitarios o preuniversitarios y capital político, tienden a desempeñarse en el sector público o a abandonar la vida pública, a diferencia de aquellos que cuentan con un nivel superior al universitario y capital familiar, que lo hacen en el privado.

Tabla 2. Capital original.

\begin{tabular}{|l|c|c|c|c|}
\hline & $\begin{array}{c}\text { Político } \\
\%(\mathbf{N})\end{array}$ & $\begin{array}{c}\text { Económico } \\
\%(\mathbf{N})\end{array}$ & $\begin{array}{c}\text { Familiar } \\
\%(\mathbf{N})\end{array}$ & $\begin{array}{c}\text { Total } \\
\%(\mathbf{N})\end{array}$ \\
\hline Público & $64,4(38)$ & $8,5(5)$ & $25,4(15)$ & $100(62)$ \\
\hline Privado & $33,3(10)$ & $6,7(2)$ & $50(15)$ & $100(26)$ \\
\hline Abandono & $64,3(18)$ & $7,1(2)$ & $28,6(8)$ & $100(30)$ \\
\hline Total & $56,4(66)$ & $7,7(9)$ & $32,5(38)$ & $100(119)$ \\
\hline
\end{tabular}

Fuente: elaboración propia.

Tabla 3. Familia en política.

\begin{tabular}{|l|c|c|c|}
\hline & $\begin{array}{c}\text { Sí } \\
\%(\mathbf{N})\end{array}$ & $\begin{array}{c}\text { No } \\
\%(\mathbf{N})\end{array}$ & $\begin{array}{c}\text { Total } \\
\%(\mathbf{N})\end{array}$ \\
\hline Público & $46,7(28)$ & $53,3(32)$ & $100(62)$ \\
\hline Privado & $60(18)$ & $40(10)$ & $100(26)$ \\
\hline Abandono & $39,3(11)$ & $60(17)$ & $100(31)$ \\
\hline Total & $48,3(57)$ & $51,7(61)$ & $100(119)$ \\
\hline
\end{tabular}

Fuente: elaboración propia.

Tabla 4. Máximo nivel de estudios.

\begin{tabular}{|l|c|c|c|c|}
\hline & $\begin{array}{c}\text { Posgrado } \\
\%(\mathbf{N})\end{array}$ & $\begin{array}{c}\text { Universitarios } \\
\%(\mathbf{N})\end{array}$ & $\begin{array}{c}\text { Preuniversitarios } \\
\%(\mathbf{N})\end{array}$ & $\begin{array}{c}\text { Total } \\
\%(\mathbf{N})\end{array}$ \\
\hline Público & $30,6(19)$ & $51,6(32)$ & $17,7(11)$ & $100(62)$ \\
\hline Privado & $65,4(17)$ & $29,6(8)$ & $3,8(1)$ & $100(26)$ \\
\hline Abandono & $22,6(7)$ & $54,8(17)$ & $22,6(7)$ & $100(31)$ \\
\hline Total & $36,1(43)$ & $47,9(57)$ & $16(19)$ & $100(119)$ \\
\hline
\end{tabular}

Fuente: elaboración propia. 
En números absolutos (véase tabla 4), de 26 individuos que se ubicaron en el sector privado, 17 tenían estudios de posgrado, 8 estudios universitarios y 1 estudios preuniversitarios. Si se incorporan variables como el sexo, la edad o la profesión, se puede corroborar que, para el caso de los expresidentes latinoamericanos, en concordancia con autores como Edurne Uriarte (1997) o Vicenzo Galasso y Tommaso Nannicini (2011), la posesión de ciertas características objetivables tiende a diferenciar a aquellos expresidentes que salen del sector público para ubicarse en la esfera privada. Si existe una tendencia a que el acceso a la élite política favorezca notablemente a hombres que pertenecen a una clase social media o alta, que han cursado estudios universitarios y en instituciones de elite, así como haber nacido en entornos urbanos (Uriarte, 1997), en el caso de los expresidentes latinoamericanos entre 1978 y 2018 solo 16\% contaban con estudios preuniversitarios. Esta diferencia se acentúa al observar que 17,7\% se desempeñó en el sector público, 22,6\% abandonó la vida pública y solo $3,8 \%$ lo hizo en la esfera privada.

Al mismo tiempo, del porcentaje marginal de mujeres que llega a la Presidencia $(5,4 \%)$, el tránsito del sector público al privado refuerza esta tendencia: de siete mujeres que han Ilegado a la Presidencia durante el periodo analizado, solo Laura Chinchilla y Violeta Barrios de Chamorro han tenido una trayectoria pospresidencial privada. Si se atiende a variables como el mecanismo de salida, se constata que de 10,3\% de los presidentes que han sido inhabilitados, solo uno se ha inclinado hacia el sector privado. Respecto de variables como la edad, 91,6\% de los presidentes con más de 70 años al dejar el cargo siguió su carrera política en el sector público, y todos los individuos que llegaron a la Presidencia con la profesión de político se ubicaron en el sector público (véase tabla 5).

Tabla 5. Trayectorias pospresidenciales: carrera política y capital político.

\begin{tabular}{|l|c|c|c|}
\hline \multicolumn{1}{|c|}{ Variable } & $\begin{array}{c}\text { Público } \\
\%(\mathbf{N})\end{array}$ & $\begin{array}{c}\text { Privado } \\
\%(\mathbf{N})\end{array}$ & $\begin{array}{c}\text { Total } \\
\%(\mathbf{N})\end{array}$ \\
\hline Inhabilitación & $91,6(11)$ & $8,4(1)$ & $10,3(12)$ \\
\hline Mujer & $71,4(5)$ & $28,5(2)$ & $5,4(7)$ \\
\hline Posibilidad reelección & $63,1(65)$ & $44,4(12)$ & $59,2(77)$ \\
\hline Más de 70 años & $91,6(11)$ & $8,4(1)$ & $10,3(12)$ \\
\hline Político de profesión & $100(9)$ & $0(0)$ & $7(9)$ \\
\hline
\end{tabular}

Fuente: elaboración propia. 
¿A dónde van los expresidentes latinoamericanos? Salida de la política y trayectorias...

Estos datos ilustran cómo la posesión de ciertos rasgos objetivables relativos a la carrera política y al capital político — genero, nivel de estudios, profesión, socialización política familiar - tiende a favorecer el acceso de un grupo minoritario de expresidentes que abandonan el sector público para ubicarse en la esfera privada (véase gráfica 2). Algunos indicadores arrojan mayor relevancia para explicar las trayectorias de aquellos que salen del sector público, dentro de los cuales sobresalen el sexo, el nivel de estudios, la profesión, el capital original o la socialización política familiar.

Gráfica 2. Análisis de correspondencias múltiples: trayectorias privadas.

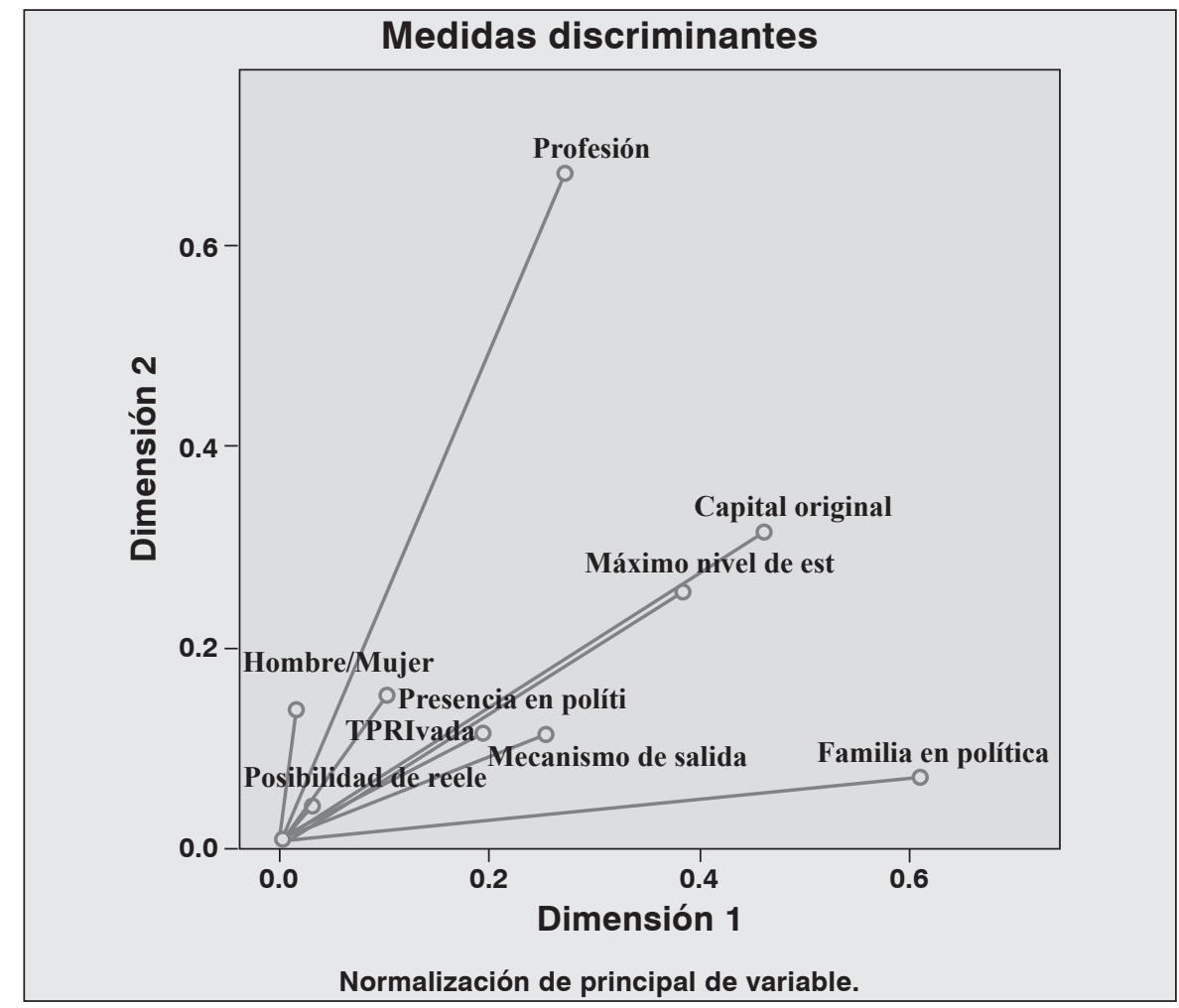

Fuente: elaboración propia.

Por otro lado, este análisis da cuenta de cómo 62 expresidentes $(52,1 \%)$ tienden a continuar con su carrera política en el sector público. De 77 individuos que tenían la posibilidad de reelegirse al dejar el cargo, $63.1 \%$ se inclinaron al sector público, lo cual sugiere que este brinda mayores posibilidades para regresar a la Presidencia. Aunque quienes se ubican en 
la esfera privada son un grupo minoritario $(21,8 \%)$, los datos dan cuenta de cómo quienes se desempeñaron en actividades mayoritariamente privadas (65\%) tienen o tuvieron presencia en política sin cargo. Este porcentaje sugiere que, a pesar de que la mayoría de los expresidentes tienden a continuar su carrera política en las instituciones públicas, hacerlo desde el sector privado no necesariamente representa un impedimento.

\section{Salida de la política: trayectorias privadas}

El grupo más reducido de expresidentes es aquel con mayoría en el sector privado. Hay casos híbridos que comparten patrones comunes a lo largo de dos dimensiones, las cuales incluyen seis tipos de actividades. Este rango comprende tanto actividades que siguen teniendo directamente vinculación política en los ámbitos nacional e internacional — desde cargos honorarios partidistas hasta funcionarios internacionales-, como aquellas que en esencia no la tienen —empresas privadas, fundaciones, consejos de administración- (véase gráfica 3).

Gráfica 3. Trayectorias privadas: dimensiones relevantes.

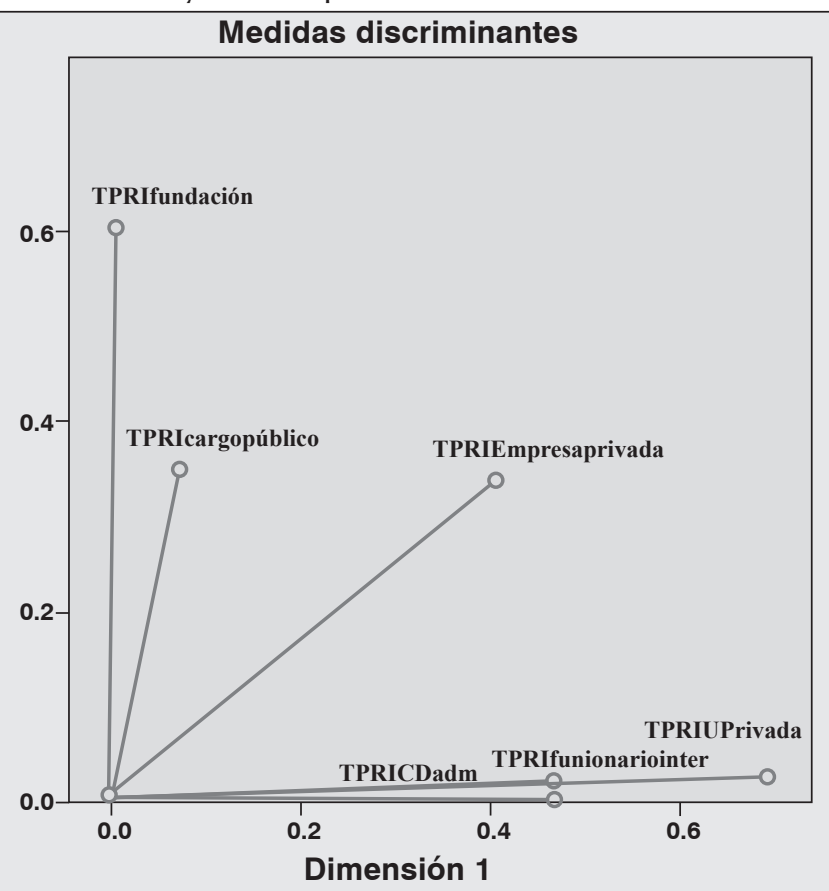

Normalización de principal de variable.

Fuente: elaboración propia. 
Dentro de estas seis actividades hay tres que tienen mayor peso en ambas dimensiones: fundaciones, empresa privada y cargos con vinculación política en el ámbito internacional. La segunda dimensión está compuesta por los expresidentes que se desempeñan en fundaciones, así como por quienes ocupan un cargo público en el ámbito nacional; y la primera, por aquellos que se desempeñan en cargos con vinculación en el ámbito supranacional, en universidades privadas y en consejos de administración.

Hay, por un lado, expresidentes que se desempeñan a tiempo completo en negocios privados. Al dejar la presidencia de Guatemala, Jorge Serrano Elías obtuvo asilo político por parte del entonces presidente de Panamá, Guillermo Endara, lo cual le permitió fungir como empresario privado con negocios relacionados con las inversiones inmobiliarias, la cría de caballos o la explotación de cantera. Hipólito Mejía, expresidente de República Dominicana, se desempeñó en actividades privadas, creando una oficina de asuntos políticos e iniciándose en la artesanía de la madera. Al dejar la Presidencia de Ecuador, Alfredo Palacio se dedicó a seguir ejerciendo como médico de forma privada. Carlos Flores Facussé, en Honduras, tuvo una significativa presencia en la esfera pública del país — la cual puede ser verificada durante el golpe de Estado que en 2009 depuso a Manuel Zelaya-, aunque se dedicó a actividades mayoritariamente privadas. ${ }^{14}$

Otro grupo de exmandatarios es el de quienes se desempeñan en consejos de administración y en fundaciones. En México, Ernesto Zedillo cuenta con una amplia trayectoria desde que dejó la presidencia en 2000 —Procter and Gamble, Alcoa, Union Pacific o la fundación Bill \& Melina Gates-; Felipe Calderón, tras dejar el cargo, creó su propia fundación y se incorporó al consejo de administración de Iberdrola, en su filial estadounidense Avangrid, donde ya se desempeñaba su exsecretaria de Energía, Georgina Kessel. Tras dejar la presidencia en 1997 y 2006, Violeta Barrios de Chamorro y Vicente Fox crearon fundaciones con sus respectivos nombres.

El tercer grupo es el de quienes compatibilizan algún cargo partidista nacional con una empresa privada o fundación. A menos de un año de haber dejado el cargo, Fernando Henrique Cardoso creó la fundación Instituto

\footnotetext{
${ }^{14}$ Fue accionista del grupo periodístico y editor del diario La Tribuna, miembro de la Asociación Nacional de Industriales de Honduras (ANDI) y del Consejo Hondureño de la Empresa Privada (COHEP).
} 


\section{Cristian Márquez Romo}

Fernando Henrique Cardoso en Sao Paulo, fungiendo como presidente honorífico del Partido de la Social Democracia Brasileña (PSDB). ${ }^{15}$ Al dejar la Presidencia de Ecuador, Osvaldo Hurtado fundó la Corporación de Estudios para el Desarrollo (CORDES) y actuó como miembro de la empresa privada de consultoría Alianza Latinoamericana de Consultorías Económicas (LAECO). Ricardo Maduro Joest, al dejar la Presidencia de Honduras, puso fin a su carrera política, dedicándose a la dirección ejecutiva de la empresa Inversores la Paz y a la Fundación para la Educación Ricardo Ernesto Maduro Andreu (Ferema).

El último grupo es el de aquellos exmandatarios que se desempeñan en universidades privadas o como funcionarios internacionales, así como quienes realizan múltiples actividades tanto en el sector privado, en fundaciones o en instituciones supranacionales. Desde que dejó la Presidencia de Costa Rica en 2014, Laura Chinchilla ha ejercido como profesora del Instituto Tecnológico de Monterrey y de la Universidad de Georgetown, desempeñándose como consultora para el Programa de las Naciones Unidas para el Desarrollo (PNUD), el Banco Interamericano de Desarrollo (BID), la Agencia de los Estados Unidos para el Desarrollo Internacional (USAID) y la Organización de los Estados Americanos (OEA). En 1988, Oscar Arias creó la Fundación

Oscar Arias para la Paz y el Progreso, presidiéndolo hasta 1996, cuando fue sucedido por su hermano, Rodrigo Arias. ${ }^{16}$ Durante su primer periodo pospresidencial, Julio María Sanguinetti (1985-1990 y 1995-2000) colaboró con la Universidad de Georgetown y presidió la fundación Instituto PAX,17 y a la fecha se desempeña como consejero de la fundación Jóvenes Líderes y de The Dialogue, entre otras. ${ }^{18}$ Por su parte, al dejar la Presidencia de Costa Rica, Figueres Olsen regresó al mundo empresarial, desempeñándose como

\footnotetext{
${ }^{15}$ Presidió, además, la Fundación de la Orquesta Sinfónica del Estado de Sao Paulo y desde el PSDB hizo oposición a Lula Da Silva y Dilma Rousseff, del Partido de los Trabajadores (PT), en distintas elecciones. ${ }^{16}$ Posteriormente, formó parte de diversos foros y organismos internacionales, como el Consejo InterAcción, la Red Internacional de Negociación, el Consejo de Presidentes y Primeros Ministros del Centro Carter, el Grupo Internacional de Crisis, el Directorio Internacional del Centro Shimon Peres por la Paz, entre otros. ${ }^{17}$ Durante este intervalo trabajó como articulista en la agencia de noticias EFE y el periódico El País. En 1991 dirigió el seminario América Latina en su laberinto, el cual impartió en la Universidad Complutense de Madrid, junto con otros seminarios y conferencias en universidades como la Internacional Menéndez y Pelayo de Santander, España, donde igualmente presidió la primera Conferencia Iberoamericana del Libro. ${ }_{18}$ Presidente honorario del Club Atlético Peñarol, doctor honoris causa de universidades como la de Brasilia, Moscú o Asunción, y forma parte del Consejo InterAcción y del Consejo de Presidentes y Primeros Ministros del Programa de las Américas del Centro Carter.
} 
¿A dónde van los expresidentes latinoamericanos? Salida de la política y trayectorias...

directivo de la empresa Digital Nations Consortium, del Instituto de Tecnología de Massachusetts (MIT). ${ }^{19}$

Finalmente, de los exmandatarios que se incorporaron a la esfera privada, la mayoría lo hizo a través de fundaciones (30,6\%), seguido por la empresa privada (26,5\%) (véase tabla 6$)$. De este grupo minoritario de exmandatarios que sale de la política, como se ha mencionado, el $65.3 \%$ han tenido presencia pública sin cargo. Esto sugiere que, pese a no tener claridad sobre las posibles consecuencias sobre el sistema político, los expresidentes tienden a seguir teniendo influencia en la esfera pública independientemente del sector en el cual se ubiquen profesionalmente. Resulta relevante, por tanto, considerar a futuro la importancia que tienen estas actividades, las opciones que ofrecen para rentabilizar el capital obtenido tras el paso por la presidencia, así como problematizar los incentivos que ofrecen a los expresidentes antes y después de dejar el cargo.

Tabla 6. Tipos de actividades privadas.

\begin{tabular}{|l|c|c|}
\hline \multicolumn{1}{|c|}{ Dimensiones } & Cantidad de casos & Porcentaje \\
\hline Fundaciones & 15 & 30,6 \\
\hline Empresa privada & 13 & 26,5 \\
\hline Funcionario internacional & 7 & 14,2 \\
\hline Universidad privada & 6 & 12,2 \\
\hline Cargo público nacional & 6 & 12,2 \\
\hline Consejo de administración & 2 & 4,3 \\
\hline Total & $\mathbf{4 9}$ & $\mathbf{1 0 0}$ \\
\hline
\end{tabular}

Fuente: elaboración propia.

\section{Conclusiones}

Los expresidentes tienden a continuar su carrera política en el sector público. En la mayoría de los países se inclinan hacia esta dimensión, que brinda mayores posibilidades para regresar a la Presidencia. No obstante, el sector privado no representa un impedimento para tener presencia política

\footnotetext{
${ }^{19}$ En 2000 se convirtió en presidente de una firma especializada en telecomunicaciones, Terremark Worldwide, y además de ser fundador del Club de Madrid, es miembro de organizaciones como el Club de Roma, la Clinton Global Initiative, AirHarbour Technologies Ltd., entre otros. Creó la Fundación Costa Rica para el Desarrollo Sostenible (Entebbe).
} 
sin cargo, por lo que los expresidentes tienden a continuar su carrera política independientemente del sector en el cual se ubiquen. ¿Qué consecuencias puede tener esto para las democracias latinoamericanas, históricamente marcadas por el presidencialismo? ¿Qué impacto puede tener esta presencia, desde uno u otro sector, en la opinión pública y en el surgimiento de nuevos liderazgos? ¿Qué diferencias hay entre los mecanismos que utilizan los expresidentes que hacen política a través de las instituciones y aquellos que se desempeñan en actividades privadas? Estas y otras cuestiones son las que quedan planteadas para futuras investigaciones.

Las trayectorias pospresidenciales sugieren la necesidad de problematizar las dimensiones para distinguir a los «políticos» de los «expolíticos», profundizando en las particularidades que explican que, con dinámicas políticas e institucionales al interior de cada país, haya ciertos elementos transversales que tienden a propiciar el acceso a un grupo minoritario de expresidentes que transitan del sector público al privado, así como las consecuencias que puede tener sobre un sistema político democrático. En aras de explorar futuras líneas de investigación, resulta de especial relevancia considerar el papel que desempeñan las fundaciones y la empresa privada en la trayectoria de los jefes de Estado, las diferencias entre países y sistemas políticos en términos de las distintas actividades privadas, el vínculo entre las élites políticas, las élites empresariales o los interlocking directorates. ¿Es necesario restringir la rentabilización del capital político?, ¿qué consecuencias tiene en términos de la calidad institucional y los mecanismos de rendición de cuentas?, ¿̇hasta qué punto es legítimo que los expresidentes continúen con su carrera política a través de otros medios? Estas preguntas abren la posibilidad de discutir, a partir de la evidencia, las transformaciones que ha tenido el presidencialismo en la región durante el presente siglo, así como las consecuencias políticas para cada uno de los países latinoamericanos, marcados históricamente por el papel de la institución presidencial.

\section{Referencias bibliográficas}

1. Alcántara, Manuel. (2012). El oficio de político. Madrid: Tecnos.

2. Alcántara, Manuel. (2013). De políticos y política: profesionalización y calidad en el ejercicio público. Perfiles Latinoamericanos, 41, pp. 19-44. https://doi. org/10.18504/pl2141-019-2013

3. Alcántara, Manuel. (2016). Los ciclos políticos en América Latina (1978-2015). Sistema: Revista de Ciencias Sociales, 242-243, pp. 5-22. 
¿A dónde van los expresidentes latinoamericanos? Salida de la política y trayectorias...

4. Alcántara, Manuel; Barragán, Mélany y Sánchez, Francisco. (2016). Los presidentes latinoamericanos y las características de la democracia. Colombia Internacional, 87, pp. 21-52. https://doi.org/10.7440/colombiaint87.2016.02

5. Alcántara, Manuel; Blondel, Jean \& Thiébault, Jean Luis. (2018). Presidents and democracy in Latin America. New York: Routledge. https://doi. org/10.4324/9781315112664

6. Alcántara, Manuel y Tagina, María Laura (eds.). (2016). Elecciones y cambio de élites en América Latina 2014-2015. Salamanca: Universidad de Salamanca.

7. Anderson, Lisa. (2010). The Ex-presidents. Journal of Democracy, 21, pp. 6578. https://doi.org/10.1353/jod.0.0166

8. Barcelona Centre for Internacional Affairs (Cidob). (s. f.). Biografías Líderes Políticos. CIDOB. Recuperado de https://www.cidob.org/biografias_lideres_politicos

9. Barragán Manjón, Mélany. (2015). El estudio de las élites parlamentarias en América Latina: pasado, presente y futuro. Revista Andina de Estudios Políticos, 5 (2), pp. 4-30.

10. Barragán Manjón, Mélany. (2016a). La salida de la política. Iberoamericana, XVı (63), pp. 227-231.

11. Barragán Manjón, Mélany. (2016b). Carreras políticas en países descentralizados. (Tesis doctoral). Universidad de Salamanca, Salamanca. Recuperado de https://gredos.usal.es/jspui/bitstream/10366/132907/1/DDPG_ Barrag\%C3\%A1nManj\%C3\%B3nM_CarrerasPol\%C3\%ADticas.pdf

12. Cabezas, Lina M. y Barragán Manjón, Mélany. (2014). Repensando la profesionalización de los políticos. Iberoamericana, 14 (54), pp. 164-168.

13. Cárdenas, Julián. (2016). Enredando a las élites empresariales en América Latina: análisis de redes de interlocking directorates y propiedad en México, Chile, Perú y Brasil. América Latina Hoy, 73, pp. 15-44. https://doi.org/10.14201/alh2016731544

14. Crabtree John \& Durand, Francisco. (2017). Peru: Elite Power and Political Capture. London: Zed.

15. Dahl, Robert. (2010). ¿Quién gobierna? Democracia y poder en una ciudad estadounidense. Madrid: Centro de Investigaciones Sociológicas.

16. Ellis, Joseph J. (2004). His Excellency. New York: Vintage.

17. Galasso, Vicenzo \& Nannicini, Tommaso. (2011). Competing on Good Politicians. American Political Science Review, 105 (1), pp. 79-99. https://doi. org/10.1017/S0003055410000535

18. Hyman, Herbert. (1959). Political Socialization. Glencoe: The Free Press.

19. Joignant, Alfredo. (2012). Habitus, campo y capital. Elementos para una teoría general del capital político. Revista Mexicana de Sociología, 4 (74), pp. 587-618.

20. Levitsky, Steven \& Way, Lucan A. (2012). Competitive Authoritarianism. Hybrid Regimes After the Cold War. Cambridge: Cambrige University.

21. Linz, Juan. (2013). Los peligros del presidencialismo. Revista Latinoamericana de Política Comparada, 7, pp. 11-31. 


\section{Cristian Márquez Romo}

22. Merino, Mauricio. (2003). México: la transición votada. América Latina Hoy, 33, pp. 63-72.

23. Mills, Charles W. (1956). The Power Elite. New York-London: Oxford University.

24. Morlino, Leonardo. (ed.) (2014). La calidad de las democracias en América Latina. San José de Costa Rica: Idea Internacional.

25. Pasquino, Gianfranco. (2011). Nuevo curso de ciencia política. México, D. F.: FCE.

26. Pérez Liñán, Aníbal. (2010). El método comparativo y el análisis de configuraciones causales. Revista Latinoamericana de Política Comparada, 3, pp. 125-148.

27. Restrepo Osorio, Luis. (1945). Democracia y dictadura en Simón Bolívar. Revista Universidad Pontificia Bolivariana, 12 (43), pp. 75.

28. Rouquié, Alan. (2011). A la sombra de las dictaduras. Buenos Aires: FCE.

29. Samuels, David J. \& Shugart, Matthew S. (2010). Presidents, Parties and Prime Ministers. How the Separation of Powers Affects Party Organization and Behavior. New York: Cambridge University. https://doi.org/10.1017/CBO9780511780882

30. Sartori, Giovanni. (1992). Elementos de teoría política. Buenos Aires: Alianza.

31. Sartori, Giovanni. (2002). La política. Lógica y método en las ciencias sociales. México, D. F.: FCE.

32. Schlesinger, Joseph A. (1966). Ambition and Politics: Political Careers in the [ 60 ] United States. Chicago: Rand McNally.

33. Schmitter, Philippe. (2010). Democracy Under Scrutiny: Elites, Citizens, Culture. European Political Science, 9 (4), pp. 511. https://doi.org/10.1057/ eps. 2010.63

34. Serrafero, Mario. (2011). La reelección presidencial indefinida en América Latina. Revista de Instituciones, Ideas y Mercados, 54, pp. 225-259.

35. Siavelis, Peter \& Morgenstern, Scott (comps.). (2008). Pathways to Power. Political Recruitment and Candidate Selection in Latin America. Pennsylvania: Pennsylvania State University.

36. Sribman Mittelman, Ariel D. (2014). La sucesión del poder. Una aproximación general desde experiencias de los siglos xx y xxı. (Tesis doctoral). Universidad de Salamanca, Salamanca.

37. Uriarte, Edurne. (1997). El análisis de las élites políticas. Revista de Estudios Políticos, 97, pp. 249-275.

38. Weber, Max. (1956). El político y el científico. Madrid: Alianza. 